\title{
Knowledge, Attitude, and Practices Towards Added Sugar Consumption among Undergraduate Female Students in Madinah, Saudi Arabia: A Cross-Sectional Study
}

Walaa A Mumena ( $\nabla$ wmumena@taibahu.edu.sa )

Taibah University https://orcid.org/0000-0003-4007-0006

Asma A Alamri

Taibah University

Alhanouf A Mahrous

Taibah University

Bushra M Alharbi

Taibah University

Jumanah S Almohaimeed

Taibah University

Maysaa I Hakeem

Taibah University

Hebah A Kutbi

King Abdulaziz University

\section{Research}

Keywords: Added sugar; obesity; underweight; dietary quality; Saudi Arabia; students

Posted Date: January 22nd, 2020

DOI: https://doi.org/10.21203/rs.2.21600/v1

License: (c) (7) This work is licensed under a Creative Commons Attribution 4.0 International License. Read Full License

Version of Record: A version of this preprint was published at Nutrition on November 1st, 2020. See the published version at https://doi.org/10.1016/j.nut.2020.110936. 


\section{Abstract}

Background: Intake of added sugar among populations in developed countries is linked to the increased prevalence of many non-communicable diseases; however, data regarding added sugar intake in developing countries are still lacking. This study aimed to evaluate knowledge, attitude, and practices towards added sugar intake and the associations with nutrient intake and weight status. Methods: This cross-sectional study was conducted among 190 healthy undergraduate female students of Taibah University, Madinah. Demographics, dietary intake, and anthropometrics (height, weight, and waist circumference (WC)) were collected during face-toface interviews. Results : Even though $55.8 \%$ of students $(n=106)$ were aware about the health consequences of excessive intake of added sugar, only $32.6 \%(n=62)$ made an effort to reduce their added sugar intake. Nine percent of students $(n=16)$ consumed carbonated soft drinks daily, while $16.1 \%$ of students $(n=30)$ consumed fruit drinks daily. Eighty-two percent $(n=155)$ of the sample exceeded the recommendation of added sugar of $<5 \%$ of total energy intake. Students who exceeded the recommendation of added sugar consumed lower quantities of protein, iron, and vitamin D. Added sugar intake was linked to nutrient intake, but not the weight status. Conclusions : Our findings indicated excessive intake of added sugar among young females. The observed inverse relationship between added sugar and macro- and micronutrient intakes highlight the urgent need for expanded efforts to limit the added sugar and to improve dietary quality of young population in Saudi Arabia.

\section{Introduction}

The dietary pattern of many populations has been shifted to be more Westernized; and the population of Arab countries, including the Saudi population, is no exception. Westernized diets are typically associated with high added sugar, salt, and fat [1]. Added sugar is the type of sugar added to foods and beverages during cooking or manufacturing [2]. Most of the added sugar consumed by Americans is coming from liquid sources, such as sugar-sweetened beverage (SSB) and fruit drinks [3]. Sugar-sweetened beverage are drinks that contain high caloric value all of which are coming from added sugar (sucrose, high fructose corn syrup, glucose, and other sweeteners), with no nutritional value $[4,5]$. Young adults were reported to be the highest consumers of SSB $[6,7]$.

Added sugar intake has been of a concern since evidence showed a negative association between added sugar intake and the intake of fresh foods, such as fruits, vegetables, and milk among adults $[8,9]$. In fact, it has been suggested that high intake of added sugar can be an indication of lower dietary quality, as the majority of high added sugar consumers tend to exceed their energy requirements in addition to consuming inadequate quantitates of a number of nutrients, including fiber, calcium, and zinc $[8,10,11]$. Additionally, excessive intake of added sugar has been linked to a number of chronic diseases, including obesity, cardiovascular diseases, and diabetes $[12,13]$. The data obtained from the National Health and Nutrition Examination Survey III reported a positive association between added sugar intake and increased risk of cardiovascular diseases mortality [14]. Furthermore, a systematic review and meta-analysis of prospective studies investigated the dietary factors of diabetes risk found a significant association between intake of SSB and diabetes risk [15].

Due to the excessive intake of added sugar among Americans and its link to lower diet quality and health problems, a number of national organizations have established recommendations of added sugar intake. For example, the Dietary Guidelines for Americans 2015-2020 recommends consuming less than 10\% of total calorie from added sugar [3], while the American Heart Association limits the amount of calories consumed from added sugar to 100 calories/d for female and 150 calorie/d for male [16]; The World Health Organization (WHO) 
has established a maximum limit of added sugar intake as an effort to reduce the intake at the global scale. The WHO recommends limiting the daily added sugar intake to less than $10 \%$ of total energy, highlighting that a further reduction to less than $5 \%$ of total energy intake would improve the health status [2]. Despite these recommendations, data reported by National Health and Nutrition Examination Survey 2011-2014 indicated high consumptions of added sugar among American adults, with an estimated average intake of $335 \mathrm{kcal} / \mathrm{day}$ in males and $239 \mathrm{kcal} /$ day in females coming from added sugar [17].

Dietary pattern and attitude of individuals towards the consumption of added sugar are proposed to be linked with several factors. For instance, the limited knowledge of what added sugar is and what are the health consequences that may result in excessive intake of added sugar. Limited knowledge of dietary sugar and sweetener classification on food labels (natural sugar, added sugar, and artificial sweetener) was evident in Ireland, as only $4 \%$ of the study sample were able to correctly classify 10 or more ingredients from a list that contained 13 items. The study also reported that only $35 \%$ of the study sample were aware of the added sugar recommendation made by the WHO [18]. Additionally, the price per calorie for foods that are high in added sugar is lower compared to the price per calorie for healthy food options [19]. In the United States, intake of added sugar was found to be higher among low-income individuals [20]. Also, another study conducted in the United States reported that individuals of lower socioeconomic and educational status tend to consume higher amount of SSB, consequently resulting in higher intake of added sugar [6]. However, the relationship between added sugar intake and socioeconomic status in high income developing countries might be very different.

As an effort towards reducing obesity and comorbidities, the WHO recommended limiting added sugar intake by taxing of sugary drinks and indicated that $20 \%$ reduction in sugary drink consumption can result from taxing sugary drinks to raise prices by $20 \%$ [21]. In the United States and recently in Saudi Arabia, taxes of all sugary drinks were raised in order to increase price per calorie and to reduce accessibility of these sugary drinks among several high risk groups, including low-income individuals and children.

Despite the effort made by the Saudi government to reduce added sugar intake, factors contributing to excessive intake are important to be identified in order to reduce the consumption of added sugar and to improve the nutritional health of individuals. Due to the lack of data available regarding added sugar intake in relation to the nutritional health of populations residing in high-income developing countries, this study aimed to evaluate knowledge, attitude, and practices towards added sugar intake and the associations with diet and weight status of young females in Madinah city.

\section{Methods}

\section{Study population}

This was a cross-sectional study of 201 healthy undergraduate female students of Taibah University, Madinah, Saudi Arabia. The sample size of this study was determined based on a standard deviation (SD) of 1.4 [22], a total width of 0.5 , a confidence level of $95 \%$, and a standardized width of the confidence interval of 0.36 . Data were collected between January and March 2019. Using a random convenience sampling method, students were recruited from different colleges in Madinah (Medicine, Dentistry, Pharmacy, Nursing, Computer Science, Business Administration, and Family Sciences). Students with medical conditions that may affect weight and food intake, pregnant students, and students who were following any special diet were excluded. 
Participants were invited to participate in the study after explaining the objectives of the study. Contact information were collected to schedule a face-to-face interview for data collection. On the day of the interview, all participants were asked to sign a consent form and to answer questions asked by the researcher to complete the study questionnaire, which was consisting of 20 items. The questionnaire collected data regarding demographics (age, major, semester level, parents-education, marital-status, number of children and income), medical history, anthropometrics (weight, height, waist circumference (WC)), dietary intake (via 24-hr dietary recall). Also, the questionnaire included questions regarding the knowledge of what is added sugar; if the participants believe they understand what added sugar is; if the participants know what are the negative health consequences resulting from consuming extensive amount of added sugar; if participants are making an effort to reduce added sugar.

\section{Anthropometric and dietary data}

Anthropometric measurements were assessed using a standardized procedure. Height was measured in centimeter by a measuring tape on straight wall shoeless, while weight was measured using a digital scale (Beurer). Body mass index (BMI) was calculated as weight in kilograms divided by squared height in meters [23]. Each student's BMI was classified based on the WHO classification into four categories: underweight $(\leq 18.5$ $\mathrm{kg} / \mathrm{m}^{2}$ ), healthy weight (18.5 and $\left.24.9 \mathrm{~kg} / \mathrm{m}^{2}\right)$, overweight $\left(25.0\right.$ and $\left.29.9 \mathrm{~kg} / \mathrm{m}^{2}\right)$, and obese $\left(\geq 30 \mathrm{~kg} / \mathrm{m}^{2}\right)$ [24]. Waist circumference was measured in centimeters at the midpoint between the last costal margin and the iliac crest by using a flexible measuring tape to assess abdominal obesity. Height and WC measurements were adjusted to the nearest $0.5 \mathrm{~cm}$. The WHO cutoff of $88 \mathrm{~cm}$ for female was used to assess WC [25].

Single 24-hour dietary recall of a weekday was collected from the total sample. Additional 24-hour dietary recall of a weekend day was collected from a subsample of 25\% (48 participants) to adjust for within person variations in order to estimate the usual intake of the students included in this study. Participants were encouraged to remember the foods that were consumed during the last 24 hours. Real life-size replicas food models were used to assess students to estimate the proper quantities of the food consumed. Dietary data were entered in Nutritics software $^{\circledR}$ (version 5.09), Dublin. When added sugar content of foods were not available in Nutritics, the number was determined from the nutrition fact label and entered into the software. Ethical approval of this study was obtained from the Ethical Committee of the College of Applied Medical Sciences at Taibah University (CLN201811).

\section{Statistical analysis}

Descriptive data were presented as means \pm standard deviations (SDs), and frequency (percent). The added sugar intake categorized into two groups based on the recommendation of $<5 \%$ or $>5 \%$ of total energy intake. Fisher's exact test was performed to test the association between the groups of added sugar intake and all categorical variables included in this study. Independent t-test was performed to test the difference in mean BMI, WC, and nutrient intake across the two added sugar groups. Variables with skewed distribution, including protein, added sugar, saturated fat, trans fat, calcium, iron, zinc, vitamin $D$, and $B_{12}$, were normalized by taking the log or square root function then t-test was performed. In cases where distributions were not normalized using the log or square root function, non-parametric tests were used to test for differences in means across the different groups. A multiple linear regression model was performed to determine the association between added sugar intake and all of the macro- (energy, protein, fat, saturated fat, trans fat, carbohydrates and fiber) and micro-nutrients intake (calcium, iron, zinc, vitamin $\mathrm{B}_{12}$, sodium, potassium and vitamin D). All regression models were adjusted for 
energy intake. Statistical tests were performed using SAS® software version 9.4 (2013, SAS Institute Inc., Cary, NC, USA). All tests were two-tailed with a significant level of $95 \%$.

\section{Results}

A total sample of 190 female participants were included in this study after excluding 11 participants, flowchart of selection criteria of participants is provided in Fig. 1. When added sugar intake was compared to the recent recommendation of $<5 \%$ of the total energy intake coming from added sugar, $81.6 \%$ of the sample $(n=155)$ exceeded the daily recommendations of added sugar. Based on the WHO criteria to define the weight status, $23.2 \%(n=44)$ of the participants were underweight with mean BMI of $16.7 \pm 1.05 \mathrm{~kg} / \mathrm{m}^{2}$, while $11.6 \%(n=22)$ were overweight with mean BMI of $27.1 \pm 1.15 \mathrm{~kg} / \mathrm{m}^{2}$, and $4.21 \%(\mathrm{n}=8)$ were obese with mean BMI of $34.3 \pm$ $3.41 \mathrm{~kg} / \mathrm{m}^{2}$. Demographic characteristics of students included in this study are described in Table 1. All demographic characteristics were similar across the different groups of added sugar intake, except age. Students who exceeded the recommendation were significantly younger. 
Table 1

Demographic characteristics of Taibah University Saudi female students by quartile of added sugar intake

\begin{tabular}{|c|c|c|c|c|}
\hline & $\begin{array}{l}\text { Added sugar intake } \\
\geq 5 \% \\
(n=155)\end{array}$ & Added sugar intake $<5 \%(n=35)$ & Total & $\mathbf{p}$ \\
\hline Age, year, mean \pm SD & $20.7 \pm 1.55$ & $21.6 \pm 1.87$ & $20.9 \pm 1.64$ & $0.006^{*}$ \\
\hline \multicolumn{5}{|l|}{ Major, n (\%) } \\
\hline Health science & $47(30.3)$ & $7(20.0)$ & $54(28.4)$ & \multirow[t]{3}{*}{0.638} \\
\hline Science & $54(34.8)$ & $13(37.1)$ & $67(35.3)$ & \\
\hline Other & $54(34.8)$ & $15(42.9)$ & $69(36.3)$ & \\
\hline \multicolumn{5}{|c|}{ Parents' education, n (\%) } \\
\hline \multicolumn{5}{|l|}{ Mother } \\
\hline$\leq$ Intermediate & $47(30.3)$ & $10(28.6)$ & $58(30.5)$ & \multirow[t]{3}{*}{0.999} \\
\hline Secondary & $39(25.2)$ & $9(25.7)$ & $49(25.8)$ & \\
\hline University & $69(44.5)$ & $16(45.7)$ & $83(43.7)$ & \\
\hline \multicolumn{5}{|l|}{ Father } \\
\hline$\leq$ Intermediate & $28(18.1)$ & $7(20.0)$ & $35(18.4)$ & \multirow[t]{3}{*}{0.431} \\
\hline Secondary & $49(31.6)$ & $7(20.0)$ & $56(29.5)$ & \\
\hline University & 78 (50.3) & $21(60.0)$ & $99(52.1)$ & \\
\hline \multicolumn{5}{|l|}{ Marital status, n (\%) } \\
\hline Single & $144(92.9)$ & $31(88.6)$ & $175(92.1)$ & 0.126 \\
\hline \multicolumn{5}{|l|}{ Income, SR, n (\%) } \\
\hline$<10000$ & $61(39.4)$ & $18(51.4)$ & 79 (41.6) & \multirow[t]{2}{*}{0.426} \\
\hline$\geq 10000$ & $94(60.6)$ & $17(48.6)$ & $111(58.4)$ & \\
\hline
\end{tabular}

Assessment of knowledge about added sugar indicated that $31.6 \%$ of participants $(n=60)$ were unable to recognize the correct definition of added sugar. Misunderstanding of the correct definition of added sugar was significantly higher among students who exceeded their recommendation $>5 \%$ of total energy intake. Furthermore, more than half of the students $(55.8 \%, n=106)$ were aware of the health consequences of consuming high quantities of added sugar. Despite of this level of awareness, only $32.6 \%$ of participants $(n=62)$ made an effort to limit their consumption of added sugar (see Table 2). 
Table 2

Knowledge and attitude regarding added sugar based on groups of added sugar intake

\begin{tabular}{|c|c|c|c|c|}
\hline & $\begin{array}{l}\text { Added } \\
\text { sugar } \\
\text { intake } \\
\geq 5 \% \\
(n=155)\end{array}$ & $\begin{array}{l}\text { Added sugar intake } \\
<5 \%(n=35)\end{array}$ & Total & p \\
\hline Misunderstanding of what added sugar is, $n(\%)$ & $42(22.1)$ & $18(9.47)$ & $\begin{array}{l}60 \\
(31.6)\end{array}$ & $0.001^{\star}$ \\
\hline Real understanding of what is added sugar, $\mathrm{n}(\%)$ & $15(7.89)$ & $5(2.63)$ & $\begin{array}{l}20 \\
(10.5)\end{array}$ & 0.290 \\
\hline $\begin{array}{l}\text { Understanding of the health consequences of } \\
\text { added sugar consumption, } n(\%)\end{array}$ & $88(46.3)$ & 18 (9.47) & $\begin{array}{l}106 \\
(55.8)\end{array}$ & 0.895 \\
\hline Effort to reduce added sugar intake, $n$ (\%) & $48(25.3)$ & $14(7.37)$ & $\begin{array}{l}62 \\
(32.6)\end{array}$ & 0.126 \\
\hline * Significant level of $95 \%$ & & & & \\
\hline
\end{tabular}

Approximately $9 \%(n=16)$ of the participants reported daily consumption of carbonated soft drinks, while $16.1 \%$ $(n=30)$ reported consumption of fruits drinks on a daily basis. Based on the analysis of dietary intakes, underweight students consumed $13.7 \%$ of their total energy intake from added sugar with mean intake of $49.5 \pm$ $48.1 \mathrm{~g} /$ day, while healthy weight participants had $12.3 \%$ of their total energy intake from added sugar with mean intake of $49.0 \pm 41.1 \mathrm{~g} /$ day. The intake of overweight students was $8.63 \%$ of their energy intake coming from added sugar with mean intake of $31.6 \pm 26.2 \mathrm{~g} / \mathrm{day}$, and obese students consumed $9.86 \%$ of their energy coming from added sugar with mean intake of $39.7 \pm 37.9 \mathrm{~g} /$ day. However, linear regression analysis showed no link between added sugar intake in relation to BMI and WC. In fact, only $3.76 \%(n=7)$ had WC $>88 \mathrm{~cm}$.

Dietary intake of all macro- and micronutrients were compared among students who consumed quantities of added sugar within the recommendation vs. students who exceeded the recommendations. The data indicated that the latter group consumed significantly lower quantities of protein, iron, and vitamin D, see Table 3. In addition, multiple linear regression analysis was performed to determine the associations between added sugar intake and all of the macro- and micronutrient intake included in the analysis, adjusting for energy intake. Results showed significant associations between added sugar intake and all nutrients included in this study except transfat and vitamin D. Carbohydrate, fat, saturated fat, calcium, and potassium were positively associated with added sugar intake, while protein, fiber, sodium, iron, zinc, and vitamin $B_{12}$ were negatively associated with added sugar intake, see Table 4. 


\begin{tabular}{|c|c|c|c|}
\hline Nutrient intake & Added sugar intake $<5 \%(n=35)$ & $\begin{array}{l}\text { Added sugar intake } \\
\geq 5 \% \\
(\mathrm{n}=155)\end{array}$ & $\mathrm{p}$ \\
\hline Energy, kcal & $1499 \pm 510$ & $1523 \pm 642$ & 0.839 \\
\hline Carbohydrate, g & $206 \pm 120$ & $195 \pm 89.2$ & 0.529 \\
\hline Protein, $\mathrm{g}$ & $57.2 \pm 20.6$ & $49.0 \pm 21.2$ & $0.042^{*}$ \\
\hline Fat, g & $52.0 \pm 24.4$ & $54.1 \pm 27.4$ & 0.681 \\
\hline Saturated fat, g & $18.2 \pm 11.3$ & $19.9 \pm 11.9$ & 0.273 \\
\hline Trans fat, $\mathrm{g}$ & $0.25 \pm 0.38$ & $4.58 \pm 52.5$ & 0.782 \\
\hline Fiber, g & $15.5 \pm 11.0$ & $12.5 \pm 8.09$ & 0.080 \\
\hline Sodium, mg & $2583 \pm 1612$ & $2274 \pm 1534$ & 0.120 \\
\hline Potassium, mg & $1483 \pm 839$ & $1340 \pm 841$ & 0.132 \\
\hline Calcium, mg & $611 \pm 386$ & $500 \pm 342$ & 0.133 \\
\hline Iron, mg & $13.3 \pm 16.9$ & $8.06 \pm 5.31$ & $0.012^{\star}$ \\
\hline Zinc, mg 8 & $5.97 \pm 7.41$ & $4.15 \pm 2.50$ & 0.168 \\
\hline Vitamin $D, \mu g$ & $6.36 \pm 14.4$ & $1.75 \pm 4.03$ & $0.027 *$ \\
\hline $\mathrm{B}_{12}, \mu \mathrm{g}$ & $1.41 \pm 1.28$ & $1.37 \pm 1.31$ & 0.404 \\
\hline \multicolumn{4}{|c|}{ * Significant level of $95 \%$} \\
\hline \multicolumn{4}{|c|}{ * Significant level of 95\% } \\
\hline
\end{tabular}




\begin{tabular}{|c|c|c|c|c|}
\hline Nutrient intake & B & SE & R-square & $\mathrm{p}$ \\
\hline Carbohydrate, g & 0.10 & 0.08 & 0.83 & $\begin{array}{l}< \\
0.000 *\end{array}$ \\
\hline Protein, g & -0.15 & 0.03 & 0.55 & $<_{0.000 *}$ \\
\hline Fat, g & 0.04 & 0.03 & 0.72 & $<_{0.000 *}$ \\
\hline Saturated fat, g & 0.04 & 0.02 & 0.47 & $\begin{array}{l}< \\
0.000 *\end{array}$ \\
\hline Trans fat, $\mathrm{g}$ & 0.06 & 0.10 & 0.01 & 0.280 \\
\hline Fiber, g & -0.04 & 0.02 & 0.27 & $\begin{array}{l}<.000^{*} \\
\end{array}$ \\
\hline Sodium, mg & -5.63 & 3.03 & 0.22 & $<.000 *$ \\
\hline Potassium, mg & 0.77 & 1.53 & 0.33 & $\begin{array}{l}< \\
0.000 *\end{array}$ \\
\hline Calcium, mg & 0.48 & 0.68 & 0.25 & $\begin{array}{l}<.000^{*} \\
\end{array}$ \\
\hline Iron, mg & -0.08 & 0.02 & 0.26 & $\begin{array}{l}< \\
0.000 *\end{array}$ \\
\hline Zinc, mg & -0.03 & 0.01 & 0.17 & $\begin{array}{l}<.000 * \\
\end{array}$ \\
\hline Vitamin $D, \mu \mathrm{g}$ & -0.03 & 0.02 & 0.02 & 0.154 \\
\hline Vitamin $B_{12}, \mu g$ & -0.002 & 0.003 & 0.06 & $0.003 *$ \\
\hline
\end{tabular}

\section{Discussion}

Assessment of dietary intake revealed high consumptions of added sugar among female students. Over half of the students included in this study recognized major health consequences of excessive intake of added sugar, but only a small proportion of the students made an effort to reduce their intake of added sugar. The limited healthy options offered on campus might explain the lower tendency of the students to reduce added sugar intake. Limited awareness of the correct definition of added sugar among the participants was also observed. A similar finding was reported among population of developed countries, including Ireland [18]. Influence of the social media on the nutritional knowledge of young adults has been suggested previously [26]; however, true definition of added sugar can be difficult to be understood through the social media.

A number of food products contain high quantities of added sugar, but data from the Dietary Guidelines for Americans 2015-2020 showed that the majority of added sugars came from liquid sources [3]. In this study, carbonated soft drinks and fruits drinks were consumed on a daily basis among students $(8.60 \%$ and $16.3 \%$, 
respectively). A study conducted among students of the University of KwaZulu-Natal reported similar findings [27]. High consumption of sugary drinks among university students might also be an indication of high availability and affordability of these drinks on campus. As high consumption of added sugar from a liquid source may increase risk of obesity [28], interventions aiming to reduce availability of such food options on campus may be effective in improving the diet quality and weight status of university students living in Saudi Arabia.

In this study, added sugar intake was negatively associated with age. Students who exceeded the recommended amount of added sugar tended to be younger. This could be an indication of improved diet quality as students get older. However, added sugar intake was not linked to income or education level. These findings are consistent with findings of a cross-sectional study conducted among undergraduate students in South Africa [27], although other studies conducted in developing countries reported a positive association between intake of added sugar and socioeconomic status [29]. On the other hand, studies performed in developed countries, including the United State and the Netherlands, found that added sugar intake was negatively associated with income and educational level $[20,22]$. The lack of the association between added sugar intake with income and educational level in this study could be due to the low variability in socioeconomic status among the majority of students. It is evident that individuals living in low and middle-income developing countries are at higher risk of consuming high quantities of added sugar as compared to individuals living in developed countries [2], while the situation in high-income developing countries might be very different.

Despite the high consumption of added sugar observed among students in this study, added sugar intake was not linked to BMI, weight status, or WC; this could be explained by the high prevalence of underweight females observed among the study sample (23.2\%).

Dietary intake of added sugar has been reported to be positively associated with energy intake, as well as poor consumption of some nutrients $[8,10]$. In this study, students who consumed higher quantities of added sugar (> $5 \%$ of total energy per day) consumed less quantities of protein, iron, and vitamin D. Additionally, the multiple regression analysis revealed a positive association between added sugar intake and energy, and nutrient intake, including carbohydrate, fat, saturated fat, calcium, and potassium. The added sugar intake was also found to be negatively associated with intakes of a number of nutrients, including protein, fiber, sodium, zinc, iron, and vitamin $B_{12}$. Similar findings were reported by several studies conducted in developed countries, including Germany and Australia $[10,11]$. These associations may indicate poor quality of diet resulting from the added sugar replacement of important nutrients in the diet, which has been reported previously [8]. Even though a significant difference in vitamin $D$ intake has been observed between students who consumed quantities of added sugar within the recommendation and those who exceeded the recommendation, the regression analysis indicated lack of association between added sugar and vitamin $D$ intake. The lack of the association might be due to low intake of vitamin $D$ among the study sample. Due to the high prevalence of vitamin $D$ deficiency in Saudi Arabia [30], fortification programs are suggested to be implemented at the national level. Previous studies conducted in developed countries has reported inverse association between calcium and added sugar intake [10, 11]. Unexpectedly, calcium intake among our sample was positively associated with added sugar intake. This association could be explained by the frequent use of milk as an ingredient in many traditional dessert recipes. In fact, compared to the RDA, students in our sample consumed lower quantities of fiber, potassium, calcium, iron, zinc, and vitamins $\mathrm{D}$ and $\mathrm{B}_{12}$, while consumption of sodium was higher than the RDA recommendation [31]. High 
consumption of sodium might be the result of frequent consumption of salted chips and snacks offered on campus as a result of the abundancy of vending machines.

This study has a number of strengths. To our knowledge, the present study was the first to evaluate the intake of added sugar among the Saudi Arabian students and investigate the association of added sugar intake with the weight status and dietary intake. However, our study is limited by its cross-sectional design, which did not allow to determine causality between added sugar, weight status, and dietary intake. Findings of this study might be generalizable to the young population in Saudi Arabia.

\section{Conclusions}

High consumption of added sugar was reported among female students in Saudi Arabia, despite the high level of awareness regarding the health consequences of excessive intake of added sugar among this group. In addition, high added sugar intake was associated with higher energy intake and lower dietary intake of many important nutrients. This indicates the need for expanded efforts to reduce added sugar intake and to improve eating habits of the young population in Saudi Arabia.

\section{List Of Abbreviations}

\begin{tabular}{ll} 
BMI & Body mass index \\
\hline FFQ & Food frequency questionnaire \\
\hline NHANES & National Health and Nutrition Examination Survey \\
\hline SD & Standard deviation \\
\hline SSB & Sugar sweetened beverage \\
\hline WC & Waist circumference \\
\hline WHO & World Health Organization
\end{tabular}

\section{Declarations}

\section{Ethics approval and consent to participate}

Ethical approval of the study was obtained from the Ethical Committee of the College of Applied Medical Sciences, Taibah University, certificate number [CLN201811]. Informed consent forms were signed by all participants before data collection.

\section{Consent for publication}

Not applicable

\section{Availability of data and materials}

The datasets used and/or analyzed during the current study are available from the corresponding author on reasonable request. 


\section{Competing interests}

The authors declare that they have no competing interests.

\section{Funding}

This research did not receive any specific grant from funding agencies in the public, commercial, or not-for-profit sectors.

\section{Authors' contributions}

WM and HK were responsible for study conception and study design. $A A, A M, B A, J A$, and $M H$ were responsible for data collection. WM, AA, AM, BA, JA, and $M H$ were responsible for data coding, cleaning. WM was responsible for analysis and interpretation of data. WM, AA, AM, BA, JA, and $\mathrm{MH}$ generated the first draft of the manuscript. WM and HK critically revised the content of the manuscript and had primary responsibility for final content. All authors read and approved the final draft of the manuscript.

\section{Acknowledgements}

Thanks are due to all the participants included in this study for their time and collaboration during the data collection. We would like to thank Fatima A. Abdulhakeem, Najwan H. Jannadi, Sara M. Aloufi, Abeer A. Bahashwan, and Shatha A. Almutairi for their contribution in data collection.

\section{References}

1. Aboul-Enein BH, Bernstein J, Neary AC. Dietary transition and obesity in selected Arabic-speaking countries: a review of the current evidence. East Mediterr Heal J. 2016;22(10).

2. WHO. WHO calls on countries to reduce sugars intake among adults and children. 2015. https://www.who.int/mediacentre/news/releases/2015/sugar-guideline/en/. Accessed 20 Sep 2019.

3. USDA. Dietary guidlines for Americans 2015-2020. Eighth. America; 2015. 54-55 p.

4. Department of Health. Sugar-sweetened beverages. 2018. http://www.health.ri.gov/healthrisks/sugarsweetenedbeverages/. Accessed 20 Sep 2019.

5. Mantzari E, Hollands GJ, Pechey R, Jebb S, Marteau TM. Impact of bottle size on in-home consumption of sugar-sweetened beverages: protocol for a feasibility and acceptability study. Pilot Feasibility Stud. 2015;1(1):1-6.

6. Han E, Powell LM. Consumption patterns of sugar sweetened beverages in the United State. J Acad Nutr Diet. 2014;113(1):43-53.

7. Al Otaibi HH. Sugar sweetened beverages consumption behavior and knowledge among university students in Saudi Arabia risk on internet banking acceptance from the user perspective. J Econ Bus Manag. 2017;5(4):173-6.

8. Kaartinen NE, Simila ME, Kanerva N, Valsta LM, Harald K, Männisto S. Naturally occurring and added sugar in relation to macronutrient intake and food consumption: results from a population-based study in adults. $J$ Nutr Sci. 2017;6:1-11.

9. CNPP. Is intake of added sugars associated with diet quality? Washington, DC; 2000. 
10. Baghurst KI, Baghurst PA, Record SJ. Demographic and nutritional profiles of people consuming varying levels of added sugars. Nutr Res. 1992;12(12):1455-65.

11. Alexy U, Sichert-Hellert W, Kersting M. Associations between intake of added sugars and intakes of nutrients and food groups in the diets of German children and adolescents. Br J Nutr. 2003;90(02):441.

12. Te L, Mallard S, Mann J. Dietary sugars and body weight: systematic review and meta-analyses of randomised controlled trials and cohort studies. BMJ. 2013;7492:1-25.

13. Harvard Health. The sweet danger of sugar. 2017. https://www.health.harvard.edu/heart-health/the-sweetdanger-of-sugar. Accessed 20 Sep 2019.

14. Yang Q, Zhang Z, Gregg EW, Flanders WD, Merritt R, Hu FB. Added sugar intake and cardiovascular diseases mortality among US adults. JAMA Intern Med. 2014;30341(4):516-24.

15. Jannasch F, Kro J, Schulze MB. Dietary patterns and type 2 diabetes: a systematic literature review and meta- analysis of prospective studies. J Nutr Nutr Epidemiol Diet. 2017;1174-82.

16. AHA. Added sugars. 2018. http://www.heart.org/en/healthy-living/healthy-eating/eat-smart/sugar/addedsugars. Accessed 20 Sep 2019.

17. Rosinger A, Herrick K, Gahche J, Park S. Sugar-sweetened beverage consumption among U.S adults, 2011 2014. Natl Cent Heal Stat. 2017;(270).

18. Tierney M, Gallagher AM, Giotis ES, Pentieva K. An online survey on consumer knowledge and understanding of added sugars. Nutrients. 2017;9(1):1-13.

19. Waterlander WE, Haas WE De, Amstel I Van, Schuit AJ, Twisk JWR, Visser M, et al. Energy density, energy costs and income - how are they related? Public Health Nutr. 2010;13(10):1599-608.

20. Ervin RB, Ogden CL. Consumption of added sugars among U. S. adults, 2005 - 2010. Natl Cent Heal Stat. 2013;(122).

21. WHO. Taxes on sugary drinks: why do it?. 2016. https://apps.who.int/iris/handle/10665/260253. Accessed 20 Sep 2019.

22. Azaïs-Braesco V, Sluik D, Maillot M, Kok F, Moreno LA. A review of total \& added sugar intakes and dietary sources in Europe. Nutr J. 2017;16(1):1-15.

23. WHO. Obesity. 2018. http://www.who.int/gho/ncd/risk_factors/obesity_text/en/. Accessed 20 Sep 2019.

24. WHO. Body mass index - BMI. 2018. http://www.euro.who.int/en/health-topics/diseaseprevention/nutrition/a-healthy-lifestyle/body-mass-index-bmi. Accessed 13 Oct 2019.

25. WHO. Waist circumference and waist-hip ratio. 2008.

http://www.who.int/nutrition/publications/obesity/WHO_report_waistcircumference_and_waisthip_ratio/en/. Accessed 26 Dec 2019.

26. Klassen KM, Douglass CH, Brennan L, Truby H, Lim MSC. Social media use for nutrition outcomes in young adults: a mixed-methods systematic review. Int J Behav Nutr Phys Act. 2018;15(70).

27. Nakhooda R, Wiles N. Consumption of added sugars among undergraduate students at a South African university and its association with BMI. South African J Clin Nutr. 2018;1-8.

28. Harvard Health. Sugary drinks. 2019. https://www.hsph.harvard.edu/nutritionsource/healthy-drinks/sugarydrinks/. Accessed 20 Sep 2019.

29. Bhurosy T, Jeewon R. Overweight and obesity epidemic in developing countries: a problem with diet, physical activity, or socioeconomic status? Sci World J. 2014;2014. 
30. Alfaris NA, Alkehayez NM, Almushawah Fl, Alnaeem AN. Vitamin D deficiency and associated risk factors in women from Riyadh , Saudi Arabia. Sci Rep. 2019;9(1):1-8.

31. IOM. Dietary Reference Intakes (DRI). 2011. http://nationalacademies.org/hmd/ /media/Files/Report Files/2019/DRI-Tables-2019/2_RDAAIVVE.pdf?la=en. Accessed 8 Jan 2020.

\section{Figures}

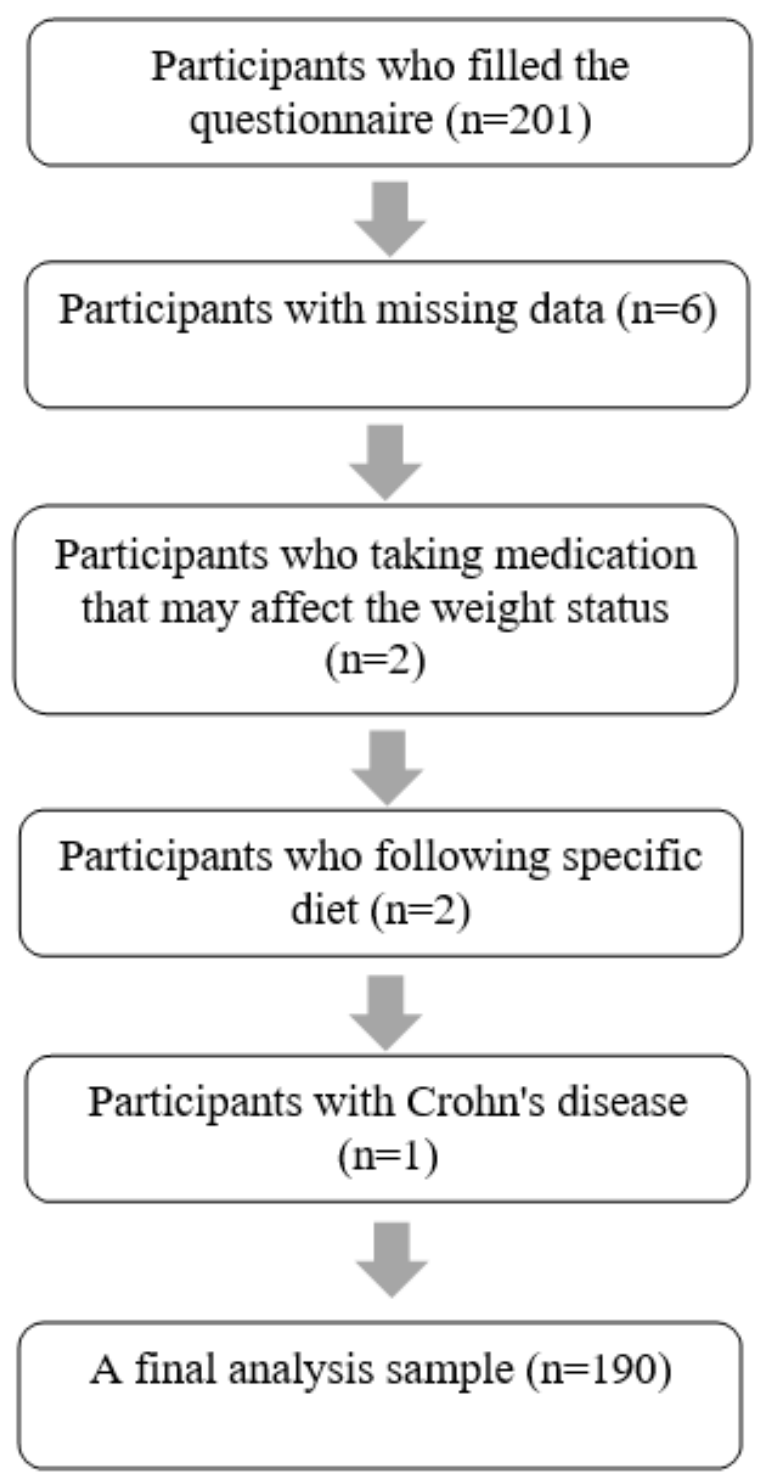

\section{Figure 1}

Flowchart of participant selection 